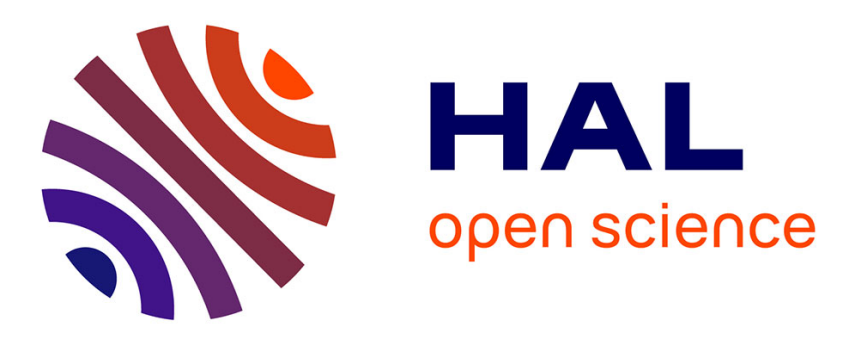

\title{
A switched control strategy including time optimal control and robust dynamic output feedback for anesthesia
}

Isabelle Queinnec, Sophie Tarbouriech, Saïd Zabi, Germain Garcia, Michel

Mazerolles

\section{To cite this version:}

Isabelle Queinnec, Sophie Tarbouriech, Saïd Zabi, Germain Garcia, Michel Mazerolles. A switched control strategy including time optimal control and robust dynamic output feedback for anesthesia. IET Control Theory and Applications, 2019, 13 (7), pp.960 - 969. 10.1049/iet-cta.2018.5260 . hal02099683

\section{HAL Id: hal-02099683 \\ https://hal.laas.fr/hal-02099683}

Submitted on 15 Apr 2019

HAL is a multi-disciplinary open access archive for the deposit and dissemination of scientific research documents, whether they are published or not. The documents may come from teaching and research institutions in France or abroad, or from public or private research centers.
L'archive ouverte pluridisciplinaire HAL, est destinée au dépôt et à la diffusion de documents scientifiques de niveau recherche, publiés ou non, émanant des établissements d'enseignement et de recherche français ou étrangers, des laboratoires publics ou privés. 


\title{
A switched control strategy including time optimal control and robust dynamic output feedback for anesthesia
}

\author{
Isabelle Queinnec ${ }^{1}$, Sophie Tarbouriech ${ }^{1}$, Saïd Zabi ${ }^{1}$, Germain Garcia ${ }^{1}$, Michel Mazerolles ${ }^{2}$ \\ ${ }^{1}$ CNRS, LAAS, Université de Toulouse, Toulouse, France, \\ ${ }^{2} \mathrm{CHU}$ Toulouse, Département d'anesthésie-réanimation, Toulouse, France \\ This paper has been published in \\ IET Control Theory \& Applications, vol. 13, issue. 7, pp. 960-969, 2019.
}

\begin{abstract}
Mimicking the medical practice, this paper deals with the control of hypnosis during a surgical intervention thanks to a switched control strategy. The key idea consists in starting with an initial minimum time control for the induction phase followed by a dynamic output feedback for the maintenance phase. The objective during the first phase is to bring the patient from its awake state to a final state corresponding to some given depth of hypnosis, measured by the BIS, within a minimum time. Then, once the patient state is close to the desired target, the control is switched to a dynamic output feedback ensuring that the BIS stays in a given interval taking into account the saturation of the actuator and the multi-time scale dynamics in the anesthesia model. Positivity of the system is also preserved thanks to the use of input saturation and state constraints. The stability of the switched control strategy is addressed and the theoretical conditions are evaluated on different case studies.
\end{abstract}

\section{Introduction}

General anesthesia consists in the control of the anesthetic and analgesic states of the patient by adjusting the perfusion of hypnotic and/or analgesic drugs based on clinical indicators such as heart rate, blood pressure and BIS (Bispectral index, derived from the spectral analysis of the electroencephalogram signal (EEG)). It therefore allows surgical intervention which, otherwise, would be very painful. The use of automatic control techniques may be considered to increase patient comfort during surgery and recovery [35], reduce dosing differences between doctors and decrease the workload of the anesthesiologists in order to minimize human error risk [24].

A general anesthesia procedure may be divided into three temporal phases: induction, maintenance and reanimation. Indeed, in a traditional anesthesia approach, the anaesthesiologist starts by an initial injection of a big amount of drugs (bolus) for a short time (induction phase), followed by a phase of manual control corresponding to the maintenance phase. The stop of the administration of anesthetic drugs marks the transition from the maintenance phase to the reanimation phase, which ends with the full resumption of consciousness and physiological functions.

Most of the works tackling the automatic control of anesthesia suggests a single control law for the two phases (induction and maintenance) using different techniques such as PID-based feedback control [1], [34], adaptive control [17] or other techniques as in [27]. The disadvantage of such approaches can be seen in the overshoot of the BIS and in the abrupt variation of the flow rate that should be avoided for the actuator. On the other hand, there exist only few works that treat each phase apart as in [14] or propose a control for either the maintenance phase [20] or the induction phase [9]. Moreover, studies generally focus on the control of one drug (hypnotic or analgesic) and we follow the same route considering only the hypnosis control of the patient. Let us point out also that the way to model the evolution of the drug in the patient body relies on the class of positive systems in the sense that state, input and output are intrinsically nonnegative at all times but only few works have been dedicated to the control problem of anesthesia in such a positive systems framework [16], [36], 
[28], [7]. It is also important to emphasize that the developments and achievements of closed-loop approaches based on the BIS indicator can be completed with other measures and/or real data as done in the literature. Actually, the clear maturity in PID or adaptive controllers methods have showed their interest during clinical tests. One can in particular promote the following works: [38], [11], [23], [19], [12], [6], [29], to cite a few among the rich literature.

The contribution of the actual paper can then be seen as a new brick in the theoretical context with the goal to complete it by considering the two phases of induction and maintenance, the clinical test being the next step. Let us emphasize that even if the framework of positive systems, and in particular the recent results [36], [13] can be attractive, we chose an alternative route in order not only to be able to formalize the complete problem combining induction and maintenance phases but also to improve the speed of convergence of the fast part of the system.

Indeed, in a previous work [40], we presented a strategy to design a robust state feedback controller for the hypnosis maintenance phase taking into account the saturation of the actuator, the multi-time scale dynamics in the anesthesia model and the variability of the patient. On the other hand, the control problem of the induction phase has been addressed in [39], using an optimal control strategy. In the current paper, the objective is to propose a full strategy mimicking the medical practice by designing a switched controller involving a first (optimal) open-loop control for the induction phase followed by a closed-loop control for the maintenance phase. It may be considered as an alternative strategy to the classical combination of a feedforward control and a feedback control that one could use to solve a tracking and regulation problem. Such a switching strategy involving successive controllers is expected to provide better performance [21].

The paper is then organized as follows: The modeling aspects and the problem formulation are presented in Section 2. Section 3 then proposes the switched control strategy and associated conditions of stability, based on the notion of set invariance. The open-loop controller is set in Section 4 and the closed-loop control design is addressed in Section 5. The approach is first illustrated in Section 6 for an ideal nominal patient model before to discuss some robustness issues closer to real life in Section 7. The paper is ended by Section 8 with concluding remarks and some perspectives for future works.

Notation. The notation throughout the paper is standard. For a vector $x$ or a matrix $A, x^{\prime}$ and $A^{\prime}$ denote the transpose of $x$ and $A$, respectively. For two symmetric matrices of the same dimensions, $A$ and $B, A>B$ means that $A-B$ is symmetric positive definite. For a matrix $A, H e(A)=A^{\prime}+A$. $I$ and 0 stand respectively for the identity and the null matrix of appropriate dimensions. For a partitioned matrix, the symbol $\star$ stands for symmetric blocks. |.| stands for the absolute value.

\section{Modeling aspects, characteristics and problem statement}

\section{$2.1 \quad$ Patient model}

Drug absorption, circulation, metabolism and elimination from the patient body are complex phenomena. However, from a control engineering point of view, compartmental mammillary models are generally considered as sufficient to represent the key ingredients of the drug dynamics in view of controller design [20]. Then, a compartment model, known as the Pharmacokinetic/Pharmacodynamic (PK/PD) model, is classically used to describe the circulation of drugs in a patient's body, as shown in Figure 1.

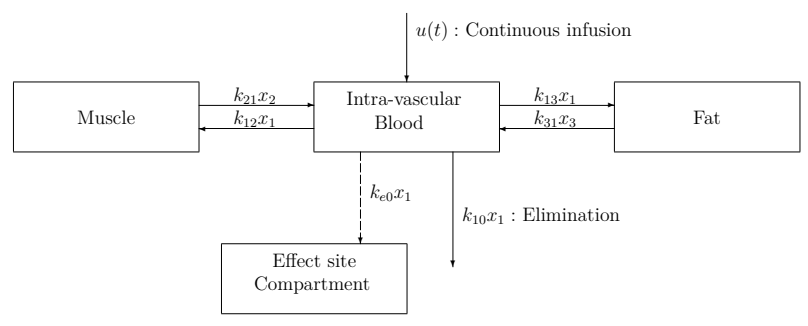

Figure 1: The compartment model

It is based on a classical three-compartment model [10] associated to a first-order dynamics to rely the 
concentration of drug in the central compartment to its action at the brain level, denoted the effect site [4]. The four-state system may then be described as follows:

$$
\dot{x}_{a n}(t)=A x_{a n}(t)+B u_{a n}(t)
$$

with

$$
\begin{aligned}
& A=\left[\begin{array}{cccc}
-\left(k_{10}+k_{12}+k_{13}\right) & k_{21} & k_{31} & 0 \\
k_{12} & -k_{21} & 0 & 0 \\
k_{13} & 0 & -k_{31} & 0 \\
k_{e 0} / v_{1} & 0 & 0 & -k_{e 0}
\end{array}\right] \\
& B=\left[\begin{array}{llll}
1 & 0 & 0 & 0
\end{array}\right]^{\prime}
\end{aligned}
$$

where $x_{a n}=\left[\begin{array}{llll}x_{a n 1} & x_{a n 2} & x_{a n 3} & x_{a n 4}\end{array}\right]^{\prime}, x_{a n 1}(t), x_{a n 2}(t), x_{a n 3}(t)$ are the masses in milligrams of the propofol in the different compartments, $x_{a n 4}$ is the effect site concentration in $\mathrm{mg} / \mathrm{l}$ and $u_{a n}$ is the infusion rate in $\mathrm{mg} / \mathrm{min}$ of the anesthetic. The parameters $k_{i j} \geq 0, \forall i \neq j, i, j=1,2,3$, are the transfer rates of the drug between compartments. The parameter $k_{10}$ represents the rate of elimination from the central compartment and $v_{1}$ corresponds to the volume of the central compartment (blood). It is customary to consider that these parameters are uncertain depending both on the inter-patient variability (i.e., the variability observed between different individuals) and intra-patient variability (i.e., the variability observed within one particular individual) [8]. Several models have been suggested in the literature to express inter-patient variability, generally distinguishing patients according to their sex, age, weight and/or size. In this paper, the Schnider model [32] is used to define simulated patients used in the numerical evaluations.

\subsection{Output indicator}

The depth of anesthesia indicator widely used by clinicians is the BIS (the bispectral index). It is a signal derived from the EEG analysis, which quantifies the level of consciousness of a patient from 0 (no cerebral activity) to typically 100 (fully awake patient). It is commonly accepted that the BIS evolution is directly related to the effect site concentration of $x_{a n 4}$, and can be described empirically by a decreasing sigmoid function [3] ${ }^{1}$ :

$$
y_{B I S}\left(x_{a n 4}(t)\right)=y_{B I S_{0}}\left(1-\frac{x_{a n 4}^{\gamma}(t)}{x_{a n 4}^{\gamma}(t)+E C_{50}^{\gamma}}\right)
$$

$y_{B I S_{0}}$ is the BIS value of an awake patient typically set to $100 . E C_{50}$ corresponds to drug concentration associated with $50 \%$ of the maximum effect and $\gamma$ is a parameter modeling the degree of non-linearity.

Remark 1 The same PK/PD model involving both the compartment dynamics and the output indicator may be viewed as a Wiener model formed with a linear block in series with a static nonlinearity as used in [25]. Delays could also be considered, as pointed out in [3], in the expression of the link between the BIS and effect site compartment, but yet considering the three-compartment mammillary model plus effect site compartment. Other approaches have considered a neural network to describe the dynamics of drugs in the body, but it does not allow to explicitly rely the model to a particular patient [42].

\subsection{Equilibrium point}

During a surgery, the BIS is generally brought then maintained close to $y_{B I S e}=50$, or at least in an interval between 40 and 60 . Given the sigmoid describing the relation between the BIS and the effect site concentration, it corresponds to the effect site concentration equal to $E C_{50}$. The values of the other variables can then be deduced from the unique equilibrium point of system (1) (see [40]):

$$
x_{e 1}=x_{4 e} v_{1}, \quad x_{e 2}=\frac{k_{12}}{k_{21}} x_{e 1}, \quad x_{e 3}=\frac{k_{13}}{k_{31}} x_{e 1}, \quad x_{e 4}=E C_{50}
$$

and the value of the associated input is given by

$$
u_{e}=k_{10} x_{e 1}
$$

\footnotetext{
${ }^{1}$ In the sequel, the time dependence is omitted to simplify the notation.
} 
Moreover, the linearized function of the BIS around this target BIS value is given by [17]:

$$
y_{B I S}=C x_{a n}+k_{b i s 0}
$$

with $C=\left[\begin{array}{llll}0 & 0 & 0 & -k_{b i s}\end{array}\right]$.

\subsection{Error model}

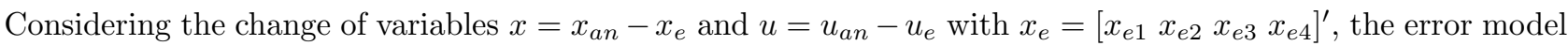
can be described as:

$$
\left\{\begin{array}{l}
\dot{x}=A x+B u \\
y=C x
\end{array}\right.
$$

The amplitude of the control is constrained as follows:

$$
U_{\min } \leq u \leq U_{\max }
$$

where $U_{\min }$ is equal to $-u_{e}$ and $U_{\max }+u_{e}$ is the maximum flow rate of the drug that can be administered in practice.

\subsection{Multi-time scale dynamics}

Regardless the patient under consideration, the dynamics of metabolism and circulation of propofol in the central compartment and at the site effect is ten times faster than in muscles, and even a hundred times faster than in fat. It can then make sense to exhibit this difference between slow and fast dynamics in the description of the system. By denoting $x_{f}=\left[\begin{array}{ll}x_{1} & x_{4}\end{array}\right]^{\prime} \in \mathbb{R}^{n_{f}}$ the fast dynamics and $x_{s}=\left[\begin{array}{ll}x_{2} & x_{3}\end{array}\right]^{\prime} \in \mathbb{R}^{n_{s}}$ the slow ones, system (5) can be written as follows:

$$
\begin{aligned}
& \dot{x}_{f}=A_{f} x_{f}+A_{f s} x_{s}+B_{f} u \\
& \dot{x}_{s}=A_{s f} x_{f}+A_{s} x_{s}
\end{aligned}
$$

with matrices $A_{f}, A_{s}, A_{f s}, A_{s f}$ and $B_{f}$ directly extracted from the original matrices $A$ and $B$ defined in (2). Similarly, the output $y$ may be written:

$$
y=C_{f} x_{f}
$$

with $C_{f}=\left[\begin{array}{ll}0 & -k_{b i s}\end{array}\right]$. Moreover, it has to be noted that the control problem is mainly related to the control of the fast dynamics as the BIS is a direct function of the concentration at the effect site and thus of the fast dynamics on which the administered drug directly acts. In that sense, the control problem may be interpreted as the control of the fast subsystem disturbed by the slow one.

\subsection{Positivity constraints}

It is important to point out that the state, the input and the output of system (1), (2), (3) are nonnegative variables and then have to respect at all times the following positivity constraints:

$$
\begin{aligned}
& x_{a n} \geq 0 \\
& u_{a n} \geq 0 \\
& y_{B I S} \geq 0
\end{aligned}
$$

Furthermore, matrix $A$ is a Metzler matrix [5]. Note that these positive constraints can be restated in the context of the error model (5) or system (7) as defined in (6) and

$$
\begin{aligned}
& x \geq-x_{e} \\
& y \geq-C x_{e}
\end{aligned}
$$

At this stage, one could feel inclined to formulate a control problem in the positive systems framework by exploiting the results developed in [33], [13]. This could be done by using a dynamic output feedback (similarly to that one defined later in (12)), in association with a Lyapunov-based stability analysis involving a diagonal 
positive matrix to build the associated quadratic Lyapunov function. In that case, according to the structure of the matrices $A, B$ and $C$ (or similarly to matrices $A_{f}, B_{f}$ and $C_{f}$ ), one can easily check that sign conditions are imposed on the matrix of the controller to preserve the positivity property for the closed-loop system. The side problem is that, with this strategy, the closed-loop dynamics cannot be improved more than the more rapid mode. It means that one can improve the slow dynamics but, as commented in Section 2.5, this is not the key problem.

Then we choose to exploit the framework associated to systems with saturated inputs [37], [41], which implicitly ensures the satisfaction of (6) and therefore implicitly the positivity constraints (9).

\subsection{Control problem formulation}

The control objective of the paper is directly inspired by the clinical practice where, in order to quickly sedate the patient and bring its state close to the equilibrium $x_{e}$, the anesthesiologist begins with a bolus injection then adjusts manually the infusion rate of the propofol to maintain the patient state close enough to the equilibrium. The control problem can then be summarized as follows:

Problem 1 Devise a control strategy embedding the induction and maintenance phases stated as two successive steps, namely an optimal open-loop control to induce quickly the hypnosis followed by a closed-loop control for the maintenance phase, and guarantee the stability of the overall switching control scheme.

\section{$3 \quad$ Stability analysis of the switched control strategy}

The key idea expressed in Problem 1 is to implement a two-step controller $u$ defined as follows:

$$
u=\left\{\begin{array}{lll}
u_{I} & \text { if } & x \in \mathbb{R}^{n} / \mathcal{D} \\
u_{M} & \text { if } & x \in \mathcal{D}
\end{array}\right.
$$

$u_{I}$ is the induction control, which corresponds to an open-loop control to bring the patient state from its awake state to the set $\mathcal{D}$ in a minimum time. $u_{M}$ is the maintenance control, which is a closed-loop control to maintain the patient state in the set $\mathcal{D}$. That means that $\mathcal{D}$ has to be an invariant set for the closed-loop system $\dot{x}=A x+B u_{M}$. The scheme in Figure 2 summarizes the approach involving the induction control phase followed by the maintenance control phase.

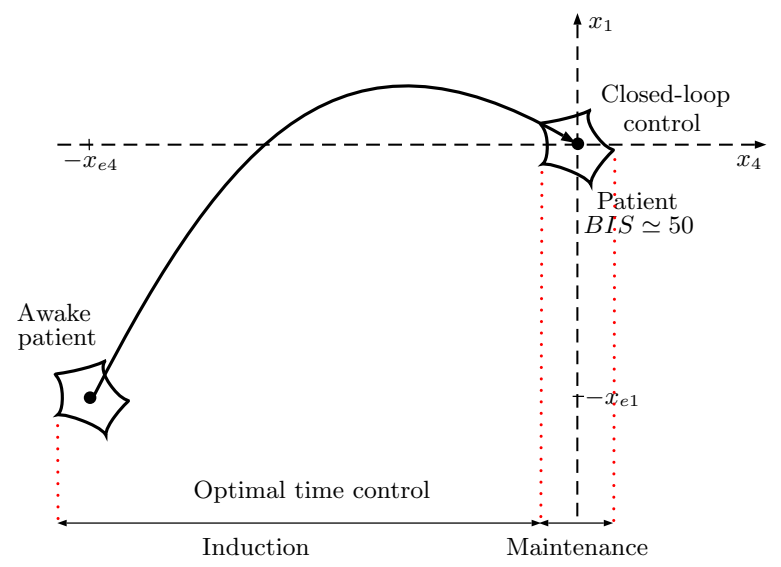

Figure 2: Scheme describing the approach with an "induction phase" followed by the "maintenance phase"

Let us consider $u_{M}$ as the saturated output of a dynamic output feedback controller of the form:

$$
\left\{\begin{array}{l}
\dot{x}_{c}=A_{c} x_{c}+B_{c} y+E_{c} \phi\left(y_{c}\right) \\
y_{c}=C_{c} x_{c}+D_{c} y
\end{array}\right.
$$


where $x_{c} \in \mathbb{R}^{n_{c}}$ is the controller state, $y \in \mathbb{R}^{p}$ is the controller input and $y_{c} \in \mathbb{R}^{m}$ its output. $A_{c}, B_{c}, C_{c}, D_{c}, E_{c}$ are given matrices of appropriate dimensions. The term $E_{c} \phi\left(y_{c}\right)$ has been added to introduce an anti-windup action to reduce the negative effects of the saturation [41], [37]. In this term, $\phi\left(y_{c}\right)=\operatorname{sat}\left(y_{c}\right)-y_{c}$ is a dead-zone function, where sat is the standard saturation function defined as:

$$
\operatorname{sat}\left(y_{c}\right)=\operatorname{sign}\left(y_{c}\right) \min \left\{\left|y_{c}\right|, u_{0}\right\}
$$

with $u_{0}$ the symmetric level of the saturation.

Remark 2 From a practical point of view, $u_{0}$ is a symmetric bound defined as $u_{0}=\min \left\{u_{e}, U_{\max }\right\}$. Considering that $U_{\max } \gg 2 u_{e}$, one can set that $u_{0}=u_{e}$.

The interconnection of system (5) with controller (12), when $u=u_{M}=s a t\left(y_{c}\right)$, gives the following closedloop system

$$
\dot{X}=\mathbf{A} X+\mathbf{B}_{\phi} \phi\left(y_{c}\right)
$$

with $X=\left[\begin{array}{ll}x^{\prime} & x_{c}^{\prime}\end{array}\right]^{\prime} \in \mathbb{R}^{\mathbf{n}}$, with $\mathbf{n}=n_{f}+n_{s}+n_{c}, y_{c}=\mathbf{K} X \in \mathbb{R}^{m}$ and

$$
\left.\begin{array}{rl}
\mathbf{A} & =\left[\begin{array}{cc}
A+B D_{c} C & B C_{c} \\
B_{c} C & A_{c}
\end{array}\right] ; \mathbf{B}_{\phi}=\left[\begin{array}{c}
B \\
E_{c}
\end{array}\right] ; \\
D_{c} C & C_{c}
\end{array}\right] \text {; }
$$

The following proposition issued from [37] gives a solution to build an ellipsoidal invariant $\operatorname{set} \mathcal{D}=\mathcal{E}\left(N^{-1}, \epsilon\right)=$ $\left\{X \in \mathbb{R}^{\mathbf{n}} ; X^{\prime} N^{-1} X \leq \epsilon^{-1}\right\}$, with a symmetric positive definite matrix $N$ and a positive scalar $\epsilon$, associated to the closed-loop system (14).

Proposition 1 If there exist a symmetric positive definite matrix $N \in \mathbb{R}^{\boldsymbol{n} \times \boldsymbol{n}}$, a diagonal positive definite matrix $S \in \mathbb{R}^{m \times m}$, a matrix $Z \in \mathbb{R}^{m \times n}$ and a positive scalar $\epsilon$ such that

$$
\begin{gathered}
{\left[\begin{array}{cc}
\boldsymbol{A} N+N \boldsymbol{A}^{\prime} & \boldsymbol{B}_{\phi} S-Z^{\prime} \\
\star & -2 S
\end{array}\right]<0} \\
{\left[\begin{array}{cc}
N & N \boldsymbol{K}_{(j)}^{\prime}-Z_{(j)}^{\prime} \\
\star & \epsilon u_{0(j)}^{2}
\end{array}\right] \geq 0 j=1, \ldots, m .}
\end{gathered}
$$

then the ellipsoid $\mathcal{E}\left(N^{-1}, \epsilon\right)$ is a region of asymptotic stability for the saturated closed loop system (14).

An implicit objective behind Problem 1 is to keep the BIS, during the maintenance phase, in an admissible interval $[40,60]$, which can be defined as a polyhedral set $\mathcal{P}$ :

$$
\mathcal{P}=\left\{X \in \mathbb{R}^{\mathbf{n}} ; a_{k}^{\prime} X \leq 1, \quad k=1, \ldots, 2\right\}
$$

Then, an optimization problem to determine the invariant set $\mathcal{D}$ may be to maximize the set included in the polyhedral set $\mathcal{P}$. It is formulated as follows:

$$
\begin{aligned}
& \min _{N, S, \epsilon} \epsilon \\
& \text { under }(15),(16), a_{k}^{\prime} N a_{k} \leq \epsilon
\end{aligned}
$$

Hence, as soon as there exists an induction control $u_{I}$ bringing $x$ in $\mathcal{D}$ from $x(0)$ in finite time, the switched control strategy (11) is solution to Problem 1 , where $\mathcal{D}$ is a solution to (17) for a given $u_{M}=\operatorname{sat}\left(y_{c}\right)$ of the form (12). 


\section{Induction control}

Let us first focus on the induction control problem. As above explained, the objective during the first phase is to bring the patient state from its awake state $x_{a n}(0)=0$ to some local set around the equilibrium target state $x_{a n}=x_{e}$ (or equivalently to bring the error model state from an initial state $x(0)=-x_{e}$ to a set $\mathcal{D}$ around the origin $x=0$ ) in a minimum time. The computation of the control that brings the system to some given set $\mathcal{D}$ in a minimum time appears to be a complicated problem. Indeed, the final state is not fixed but has to belong to a certain set, or at least to the boundary of this set. The computation of the optimal control strongly depends on this set.

An alternative route consists in taking the origin of system (5), $x=0$, as the final state, i.e, searching the minimal time control which allows to bring back the state to the origin. Then, as soon as the system trajectories enter the set $\mathcal{D}$, one can switch to the maintenance control. Moreover, as above commented, the BIS is directly related to the fast state $x_{f}$. Trying to bring the slow state $x_{s}$ to the origin would take a considerable time with no direct effect on the BIS. Thus, the strategy adopted in this paper consists in designing an optimal control that brings the fast state to the origin in a minimum time regardless of the slow ones.

The induction problem can then be written as follows:

$$
\begin{array}{ll}
\min _{u} & J(x, u)=\int_{0}^{T_{f}} d t=T_{f} \\
\text { s. } \mathrm{t} & \dot{x}=A x+B u, \quad x(0)=-x_{e}, x_{f}\left(T_{f}\right)=0 \\
& U_{\min } \leq u \leq U_{\max }
\end{array}
$$

Remark 3 As the optimal control problem (18) only ensures that $x_{f}$ goes to 0, it has to be checked that all the state enters $\mathcal{D}$ before to switch to the maintenance control. This may be done by adding a constraint in the optimization problem (17) to ensure that the slow part of the initial state $\left(x_{s}(0)=\left[-x_{e 3}-x_{e 4}\right]^{\prime}\right)$ belongs to $\mathcal{D}$ when the fast state $x_{f}$ approaches its equilibrium. This is typically done with the condition:

$$
\left[\begin{array}{cc}
\epsilon & \epsilon c_{x} \\
\epsilon c_{x} & N
\end{array}\right]>0 \text { with } c_{x}=\left[\begin{array}{lll}
0 & x_{s}(0)^{\prime} & 0
\end{array}\right]^{\prime}
$$

The minimum time control problem has been extensively studied in the literature (see [22], [2] for recent references on the subject). In the case of linear systems, the existence and uniqueness of the solution is known to depend on the controllability property of the system. The optimal control consists of a countable number of commutations between $U_{\max }$ and $U_{\min }$. Moreover, as soon as the eigenvalues of the model are real, the number of switches is at most equal to $n-1$, where $n$ is the order of the system. Hence, in our case, at most three commutations have to be considered.

The characterisation of the optimal control $u^{*}$ is detailed in [39]. Denote $\lambda \in \mathbb{R}^{n}$ a co-state vector used to define the Hamiltonian $H$ associate to problem (18):

$$
H(t, x, u, \lambda)=1+\lambda^{\prime}(A x+B u)
$$

Using the maximum principle of Pontryagin [22], it may be shown that the optimal control $u^{*}$ has a bang-bang form described by the following function:

$$
u^{*}= \begin{cases}U_{\min } & \text { if } \lambda^{*^{\prime}} B>0 \\ U_{\max } & \text { if } \lambda^{*^{\prime}} B<0 \\ \text { undetermined } & \text { if } \lambda^{*^{\prime}} B=0\end{cases}
$$

where $u^{*}$ and $\lambda^{*}$ verify the Pontryagin conditions [22]. The existence, uniqueness and normality (no indeterminability) of this control is guaranteed thanks to the fact that system (5) is fully controllable [26]. Moreover, according to the particular structure of $B$ defined in (2), function (21) can be written as

$$
u^{*}= \begin{cases}U_{\min } & \text { if } \lambda_{1}^{*}>0 \\ U_{\max } & \text { if } \lambda_{1}^{*}<0\end{cases}
$$

where $\lambda_{1}^{*}$ is the first component of vector $\lambda^{*}$. 
From a practical point of view, the initial values of the co-state vector $\lambda^{*}$ being unknown, an iterative approach has to be used [39]. On the other hand, considering that the Hamiltonian should be equal to zero at any point of the optimal trajectory and that $u\left(t=0^{+}\right)=U_{\max }$ (such that the trajectory is increasing from $x(0)=-x_{e}<0$ to $\left.x_{f}\left(T_{f}\right)=0\right)$, it follows that:

$$
\lambda_{1}^{*}(0)=\frac{-1}{U_{\max }-U_{\min }}
$$

A Newton gradient method may then be employed to determine the other components of $\lambda^{*}(0)$.

\section{Maintenance control}

\subsection{Controller description and problem formulation}

Let us turn now to the design of the maintenance control $u_{M}$, considering a dynamic output controller of the form (12). Paying attention to the multi-time scale of dynamics as described in Section 2.5 and to the particular structure of $B$ (defined in (2)), it is suggested to shift the control problem of system (5) to that one of the fast dynamics system (7a). Several cases may be considered to take care of the slow state $x_{s}$ appearing in the fast dynamics system as some additive disturbance. The simplest case may be to forget this disturbance in the design step then to evaluate the controller obtained for the overall system thanks to Proposition 1 . A more elegant option is to explicitly consider the interconnection of the two subsystems. The slow state $x_{s}$ is then considered as a bounded disturbance for the fast system belonging to some a priori given set. Similarly the fast state plays the role of a bounded disturbance for the slow subsystem $(7 \mathrm{~b})$.

The interconnection of system (7)-(8) with controller (12), when $u=u_{M}=\operatorname{sat}\left(y_{c}\right)$, may then be written as

$$
\begin{aligned}
\dot{\tilde{x}} & =\tilde{A} \tilde{x}+\tilde{B}_{\phi} \phi\left(y_{c}\right)+\tilde{B}_{s} x_{s} \\
\dot{x}_{s} & =A_{s} x_{s}+\tilde{B}_{x} \tilde{x} \\
y_{c} & =\tilde{K} \tilde{x}
\end{aligned}
$$

where

$$
\begin{aligned}
& \tilde{x}=\left[\begin{array}{l}
x_{f} \\
x_{c}
\end{array}\right] ; \tilde{A}=\left[\begin{array}{cc}
A_{f}+B_{f} D_{c} C_{f} & B_{f} C_{c} \\
B_{c} C_{f} & A_{c}
\end{array}\right] ; \tilde{B}_{\phi}=\left[\begin{array}{l}
B_{f} \\
E_{c}
\end{array}\right] \\
& \tilde{B}_{s}=\left[\begin{array}{c}
A_{f s} \\
0
\end{array}\right] ; \tilde{K}=\left[\begin{array}{ll}
D_{c} C_{f} & C_{c}
\end{array}\right] ; \tilde{B}_{x}=\left[\begin{array}{ll}
A_{s f} & 0
\end{array}\right]
\end{aligned}
$$

The maintenance control design problem may then be formulated as follows:

Problem 2 Design $A_{c}, B_{c}, C_{c}, D_{c}, E_{c}$ and characterize two invariant sets in which the trajectories of the fast and slow subsystems remain confined, respectively.

\subsection{Controller synthesis}

The solution presented in this section relies on the following result.

Proposition 2 If there exist two symmetric positive definite matrices $P \in \mathbb{R}^{\left(n_{f}+n_{c}\right) \times\left(n_{f}+n_{c}\right)}, Q \in \mathbb{R}^{n_{s} \times n_{s}}, a$ diagonal positive definite matrix $T \in \mathbb{R}^{m \times m}$, a matrix $G \in \mathbb{R}^{m \times\left(n_{f}+n_{c}\right)}$, matrices $A_{c}, B_{c}, C_{c}, D_{c}, E_{c}$ and six positive scalars $\tau_{1}, \tau_{2}, \tau_{3}, \tau_{4}, \eta$ and $\delta$ satisfying

$$
\begin{aligned}
& {\left[\begin{array}{ccc}
\tilde{A}^{\prime} P+P \tilde{A}+\tau_{1} P & P \tilde{B}_{\phi}-G^{\prime} T & P \tilde{B}_{s} \\
\star & -2 T & 0 \\
\star & \star & -\tau_{2} Q
\end{array}\right]<0} \\
& {\left[\begin{array}{cc}
A_{s}^{\prime} Q+Q A_{s}+\tau_{3} Q & Q \tilde{B}_{x} \\
\star & -\tau_{4} P
\end{array}\right]<0}
\end{aligned}
$$




$$
\begin{gathered}
{\left[\begin{array}{cc}
P & \tilde{K}_{(j)}^{\prime}-G_{(j)}^{\prime} \\
\star & \eta u_{0(j)}^{2}
\end{array}\right] \geq 0 j=1, \ldots, m .} \\
\tau_{2} \eta-\tau_{1} \delta \leq 0 \\
-\tau_{3} \eta+\tau_{4} \delta \leq 0
\end{gathered}
$$

then matrices $A_{c}, B_{c}, C_{c}, D_{c}, E_{c}$ and sets $\mathcal{E}(P, \eta):=\left\{\tilde{x} \in \mathbb{R}^{n_{f}+n_{c}} ; \tilde{x}^{\prime} P \tilde{x} \leq \eta^{-1}\right\}$ and $\mathcal{E}(Q, \delta):=\left\{x_{s} \in\right.$ $\left.\mathbb{R}^{n_{s}} ; x_{s}^{\prime} Q x_{s} \leq \delta^{-1}\right\}$ are solution to Problem 2 .

Proof: The proof mimics that one of Proposition 1 in [40] dedicated to the static state feedback case. Consider for the fast closed-loop subsystem (24a), the Lyapunov quadratic function $V(\tilde{x})=\tilde{x}^{\prime} P \tilde{x}, P=P^{\prime}>0$. We aim at proving that $\dot{V}(\tilde{x})<-\alpha(V(\tilde{x})), \alpha$ being a $\mathcal{K}$-function, for any $\tilde{x}$ such that $\tilde{x} \notin \operatorname{int}(\mathcal{E}(P, \eta)$, for any $x_{s} \in \mathcal{E}(Q, \delta)$. In other words, the following inequality must be verified using the S-procedure:

$$
\dot{V}(\tilde{x})+\tau_{1}\left(\tilde{x}^{\prime} P \tilde{x}-\eta^{-1}\right)+\tau_{2}\left(\delta^{-1}-x_{s}^{\prime} Q x_{s}\right)<-\alpha(V(\tilde{x}))
$$

We use also the modified sector condition [37], which expresses that for any $\tilde{x}$ belonging to the polyhedron $S\left(|\tilde{K}-G|, u_{0}\right)$ defined by (recall that $u_{0}$ is defined in Remark 2):

$$
S\left(|\tilde{K}-G|, u_{0}\right)=\left\{\tilde{x} \in \mathbb{R}^{n_{f}+n_{c}} ;-u_{0} \leq(\tilde{K}-G) \tilde{x} \leq u_{0}\right\}
$$

the sector condition $\phi(\tilde{K} \tilde{x})^{\prime} T(\phi(\tilde{K} \tilde{x})+G \tilde{x}) \leq 0$ is verified, $T$ being a positive diagonal matrix. Thus, a sufficient condition to verify $(30)$ is that

$$
\begin{aligned}
\dot{V}(\tilde{x})+ & \tau_{1} \tilde{x}^{\prime} P \tilde{x}-\tau_{2} x_{s}^{\prime} Q x_{s} \\
& -2 \phi(K \tilde{x})^{\prime} T(\phi(\tilde{K} \tilde{x})+G \tilde{x})<-\alpha(V(\tilde{x}))
\end{aligned}
$$

and

$$
-\tau_{1} \eta^{-1}+\tau_{2} \delta^{-1}<0
$$

as long as $\mathcal{E}(P, \eta) \subseteq S\left(|K-G|, u_{0}\right)$, which is ensured by satisfying the inequality (27). The inequality (31) can be written as

$$
\zeta^{\prime} \Omega \zeta<-\alpha(V(\tilde{x}))
$$

with $\zeta=\left[\begin{array}{lll}\tilde{x}^{\prime} \phi\left(y_{c}\right)^{\prime} & x_{s}^{\prime}\end{array}\right]^{\prime}$ and $\Omega$ being the left-hand side of inequality (25). The satisfaction of relation (25) means that $\zeta^{\prime} \Omega \zeta<0$. Therefore there exists a small enough positive scalar $\alpha$ such that $\zeta^{\prime} \Omega \zeta<-\alpha \tilde{x}^{\prime} \tilde{x}$, and thus (33) holds. Then, the satisfaction of (25), (27) and (28) guarantees the invariance of $\mathcal{E}(P, \eta)$ for the closed-loop fast subsystem, for any $x_{s} \in \mathcal{E}(Q, \delta)$. Conversely, the satisfaction of the conditions (26) and (29) guarantees the invariance of $\mathcal{E}(Q, \delta)$ for the slow subsystem, for any $\tilde{x} \in \mathcal{E}(P, \eta)$.

Proposition 2 gives a sufficient condition in terms of matrix inequalities to solve Problem 2. Nevertheless, the inequalities are nonlinear because they include products between decision variables, involving in particular matrices $A_{c}, B_{c}, C_{c}, D_{c}, E_{c}$ and $P$. In order to linearize at least partially the conditions of Proposition 2, we consider that we want to design a controller of the same order as the fast subsystem: then we consider $n_{c}=n_{f}$. Furthermore, we use the congruence transformation [31] to establish a sufficient set of conditions to solve Problem 2.

The following proposition gives constructive conditions for the dynamic output feedback controller design Problem 2.

Proposition 3 Suppose that there exist symmetric positive definite matrices $X \in \mathbb{R}^{n_{f} \times n_{f}}, Y \in \mathbb{R}^{n_{f} \times n_{f}}, M_{1} \in$ $\mathbb{R}^{n_{f} \times n_{f}}, M_{2} \in \mathbb{R}^{n_{f} \times n_{f}}, Q \in \mathbb{R}^{n_{s} \times n_{s}}$, a diagonal positive definite matrix $S \in \mathbb{R}^{m \times m}$, matrices $L \in \mathbb{R}^{m \times n_{f}}$, $F \in \mathbb{R}^{n_{f} \times p}, W \in \mathbb{R}^{n_{f} \times n_{f}}, Z_{1} \in \mathbb{R}^{m \times n_{f}}, Z_{2} \in \mathbb{R}^{m \times n_{f}}, Q_{1} \in \mathbb{R}^{n_{f} \times m}, D_{c} \in \mathbb{R}^{m \times p}, K \in \mathbb{R}^{n_{f} \times n_{f}}$ and positive scalars $\alpha, \tau_{1}, \tau_{2}, \tau_{3}, \tau_{4}, \eta$ and $\delta$ satisfying

$$
\left[\begin{array}{cccccc}
H e\left(\mathcal{L}+\frac{\tau_{1}}{2}\right) & \star & \star & \star & \star & \star \\
W^{\prime}+\mathcal{A}^{\prime}+\tau_{1} I & H e\left(\mathcal{H}+\frac{\tau_{1}}{2} X\right) & \star & \star & \star & \star \\
S B_{f}^{\prime}-Z_{1} & Q_{1}^{\prime}-Z_{2} & -2 S & \star & \star & \star \\
A_{f s}^{\prime} & A_{f s}^{\prime} X & 0 & -\tau_{2} Q & \star & \star \\
0 & X & 0 & 0 & -M_{1} & \star \\
\mathcal{L}+K & 0 & 0 & 0 & 0 & -M_{2}
\end{array}\right]<0
$$




$$
\begin{aligned}
& M_{1}+M_{2}<2 I \\
& {\left[\begin{array}{ccccc}
A_{s}^{\prime} Q+Q A_{s}+\tau_{3} Q & \star & \star & \star & \star \\
0 & -\tau_{4} Y & \star & \star & \star \\
A_{s f}^{\prime} Q & -\tau_{4} I & -\tau_{4} X & \star & \star \\
0 & A_{s f} Y & 0 & -\alpha I & \star \\
\alpha Q & 0 & 0 & 0 & -\alpha I
\end{array}\right]<0,} \\
& {\left[\begin{array}{ccc}
Y & \star & \star \\
I & X & \star \\
L_{(j)}-Z_{1(j)} & D_{c(j)} C_{f}-Z_{2(j)} & \eta u_{0(j)}^{2}
\end{array}\right] \geq 0, j=1, \ldots, m} \\
& \tau_{2} \eta-\tau_{1} \delta \leq 0 \\
& -\tau_{3} \eta+\tau_{4} \delta \leq 0
\end{aligned}
$$

with $\mathcal{A}=A_{f}+B_{f} D_{c} C_{f}, \mathcal{L}=A_{f} Y+B_{f} L$ and $\mathcal{H}=X A_{f}+F C_{f}$. Then, the dynamic output feedback (12) with matrices:

$$
\begin{aligned}
& D_{c} \\
& C_{c}=\left(L-D_{c} C_{f} Y\right)\left(V^{\prime}\right)^{-1} \\
& B_{c}=U^{-1}\left(F-X B_{f} D_{c}\right) \\
& A_{c}=U^{-1}\left(W^{\prime}+X K+F C_{f} Y+X B_{f} D_{c} C_{f} Y\right)\left(V^{\prime}\right)^{-1} \\
& E_{c}=U^{-1}\left(Q_{1}-X B_{f} S\right) S^{-1}
\end{aligned}
$$

where $U$ and $V$ are nonsingular matrices such that $U V^{\prime}=I-X Y$ and

$$
P=\left[\begin{array}{cc}
X & U \\
U^{\prime} & \hat{X}
\end{array}\right], \quad \hat{X}=U^{\prime}\left(X-Y^{-1}\right)^{-1} U
$$

and the sets $\mathcal{E}(P, \eta)$ and $\mathcal{E}(Q, \delta)$, defined as in Proposition 2, are solution to Problem 2.

Proof : Consider matrix $P$ defined in (41), then one can define its inverse $P^{-1}$ as follows

$$
P^{-1}=\left[\begin{array}{cc}
Y & V \\
V^{\prime} & \hat{Y}
\end{array}\right]
$$

for which the following relations hold

$$
\begin{aligned}
X Y+U V^{\prime} & =I & & U^{\prime} V+\hat{X} \hat{Y}=I \\
U^{\prime} Y+\hat{X} V^{\prime} & =0 & & X V+U \hat{Y}=0
\end{aligned}
$$

Define

$$
\mathcal{J}=\left[\begin{array}{ll}
Y & V \\
I & 0
\end{array}\right]
$$

which is nonsingular by construction. Pre- and post-multiplying (25) respectively by $\operatorname{diag}\{\mathcal{J}, S, I\}$ and $\operatorname{diag}\left\{\mathcal{J}^{\prime}, S, I\right\}$, with $S=T^{-1}$, it follows

$$
\left[\begin{array}{ccc}
\mathcal{J}\left(P \tilde{A}+\tilde{A}^{\prime} P+\tau_{1} P\right) \mathcal{J}^{\prime} & \star & \star \\
S \tilde{B}_{\phi}^{\prime} P \mathcal{J}^{\prime}-G^{\prime} \mathcal{J}^{\prime} & -2 S & \star \\
\tilde{B}_{s}^{\prime} P \mathcal{J}^{\prime} & 0 & -\tau_{2} Q
\end{array}\right]<0
$$

By replacing in (44) matrices $A_{c}, B_{c}, C_{c}, D_{c}$ and $E_{c}$ by their expressions given in (40), relation (44) can be written as follows

$$
\mathcal{Z}_{0}+U_{1}^{\prime} U_{2}+U_{2}^{\prime} U_{1}<0
$$

with

$$
\begin{aligned}
\mathcal{Z}_{0} & =\left[\begin{array}{cccc}
\mathcal{L}+\mathcal{L}^{\prime}+\tau_{1} Y & \star & \star & \star \\
W^{\prime}+\mathcal{A}^{\prime}+\tau_{1} I & \mathcal{H}+\mathcal{H}^{\prime}+\tau_{1} X & \star & \star \\
S B_{r}^{\prime}-Z_{1} & Q_{1}^{\prime}-Z_{2} & -2 S & \star \\
A_{f s}^{\prime} & A_{f s}^{\prime} X & 0 & -\tau_{2} Q
\end{array}\right] \\
U_{1} & =\left[\begin{array}{ccc}
X & 0 & 0
\end{array}\right] \\
U_{2} & =\left[\begin{array}{cccc}
0 & &
\end{array}\right]
\end{aligned}
$$


and with $G=\left[\begin{array}{ll}G_{1} & G_{2}\end{array}\right] ; Z_{1}=G_{1} Y+G_{2} V^{\prime} ; Z_{2}=G_{1}, Q_{1}=X B_{r} S+U E_{c} S$. By taking into account the definition of $\mathcal{Z}_{0}, U_{1}$ and $U_{2}$ given in (46), relation (34) can also be written as follows:

$$
\left[\begin{array}{ccc}
\mathcal{Z}_{0} & \star & \star \\
U_{1} & -M_{1} & \star \\
U_{2} & 0 & -M_{2}
\end{array}\right]<0
$$

which is equivalent by using the Schur complement to

$$
\mathcal{Z}_{0}+\left[\begin{array}{ll}
U_{1}^{\prime} & U_{2}^{\prime}
\end{array}\right]\left[\begin{array}{cc}
M_{1}^{-1} & \star \\
0 & M_{2}^{-1}
\end{array}\right]\left[\begin{array}{l}
U_{1} \\
U_{2}
\end{array}\right]<0
$$

By combining relation (35) with the properties $\left(I-M_{1}\right) M_{1}^{-1}\left(I-M_{1}\right) \geq 0$ and $\left(I-M_{2}\right) M_{2}^{-1}\left(I-M_{2}\right) \geq 0$, one gets $M_{1}^{-1} \geq M_{2}$ and $M_{2}^{-1} \geq M_{1}$, respectively. From this, it follows that

$$
\left[\begin{array}{cc}
M_{1}^{-1} & \star \\
-I & M_{2}^{-1}
\end{array}\right]>0
$$

Then, one gets:

$$
\begin{gathered}
\mathcal{Z}_{0}+U_{1}^{\prime} U_{2}+U_{2}^{\prime} U_{1} \\
<\mathcal{Z}_{0}+\left[\begin{array}{ll}
U_{1}^{\prime} & U_{2}^{\prime}
\end{array}\right]\left[\begin{array}{cc}
M_{1}^{-1} & \star \\
0 & M_{2}^{-1}
\end{array}\right]\left[\begin{array}{c}
U_{1} \\
U_{2}
\end{array}\right]
\end{gathered}
$$

Hence, one can conclude that if relation (34) holds, then (25) is satisfied.

By pre- and post-multiplying relation (26) by $\operatorname{diag}\{I, \mathcal{J}\}$ and $\operatorname{diag}\left\{I, \mathcal{J}^{\prime}\right\}$, respectively, one obtains:

$$
\left[\begin{array}{ccc}
A_{s}^{\prime} Q+Q A_{s}+\tau_{3} Q & \star & \star \\
Y A_{s f}^{\prime} Q & -\tau_{4} Y & \star \\
A_{s f}^{\prime} Q & -\tau_{4} I & -\tau_{4} X
\end{array}\right]<0,
$$

The left-hand side of (51) can be written also as follows

$$
\begin{aligned}
\mathcal{Z}_{1}= & {\left[\begin{array}{ccc}
A_{s}^{\prime} Q+Q A_{s}+\tau_{3} Q & \star & \star \\
0 & -\tau_{4} Y & \star \\
A_{s f}^{\prime} Q & -\tau_{4} I & -\tau_{4} X
\end{array}\right] } \\
& +H e\left(\left[\begin{array}{c}
0 \\
Y A_{s f}^{\prime} \\
0
\end{array}\right]\left[\begin{array}{lll}
Q & 0 & 0
\end{array}\right]\right)
\end{aligned}
$$

which satisfies:

$$
\begin{gathered}
\mathcal{Z}_{1} \leq\left[\begin{array}{ccc}
A_{s}^{\prime} Q+Q A_{s}+\tau_{3} Q & \star & \star \\
0 & -\tau_{4} Y & \star \\
A_{s f}^{\prime} Q & -\tau_{4} I & -\tau_{4} X
\end{array}\right] \\
+\alpha^{-1}\left[\begin{array}{c}
0 \\
Y A_{s f}^{\prime} \\
0
\end{array}\right]\left[\begin{array}{lll}
0 & A_{s f} Y & 0
\end{array}\right]+\alpha\left[\begin{array}{c}
Q \\
0 \\
0
\end{array}\right]^{\prime}\left[\begin{array}{lll}
Q & 0 & 0
\end{array}\right]
\end{gathered}
$$

for some positive scalar $\alpha$. By using the Schur complement on the right-hand side of (53), one gets the left-hand side of relation (36). It follows that if relation (36) holds then relation (51) holds and therefore relations (26) is also satisfied.

Finally relation (37) is derived by pre- and post-multiplying relation (27) by $\operatorname{diag}\{\mathcal{J}, I\}$ and $\operatorname{diag}\left\{\mathcal{J}^{\prime}, I\right\}$, respectively.

\subsection{Numerical issues}

The conditions of Proposition 3 still contain some products between the decision variables and cannot be formally considered as LMIs. Most of the nonlinearities concern the product between a scalar $\tau_{i}, i=1, \cdots, 4$ and a 
matrix (or scalar) and come from the use of the S-procedure. Although their impact on the solution cannot be neglected, it is rather common to select them such as to find a feasible solution, either with some trial-and-error procedure or with a grid search. Moreover, $\tau_{1}$ acts as a pole placement constraint on the closed-loop system and may be selected as such. Parameter $\alpha$ is used to break the nonlinear term $Q A_{s f} Y$. It has also to be selected a priori such as to find a feasible solution. The optimization may then be to enlarge the sets $\mathcal{E}(P, \eta)$ and $\mathcal{E}(Q, \delta)$ thanks for example to the parameters $\eta$ and $\delta$, by solving the optimization problem:

$$
\begin{aligned}
& \min \delta+\eta \\
& \text { under }(34)-(39)
\end{aligned}
$$

The design step performed thanks to Proposition 3 must be followed by an analysis step (Proposition 1) to make more accurate the determination of the full ellipsoidal invariant set $\mathcal{D}$.

\section{Numerical illustration}

Let us consider a patient whose characteristics are: male, 53 years, $177 \mathrm{~cm}$ and $77 \mathrm{~kg}$. The associated pharmacokinetic parameters are computed with the Schnider model [32], allowing to define:

$$
A=\left[\begin{array}{rrrr}
-0.9170 & 0.0683 & 0.0035 & 0 \\
0.3021 & -0.0683 & 0 & 0 \\
0.1958 & 0 & -0.0035 & 0 \\
0.1068 & 0 & 0 & -0.4560
\end{array}\right]
$$

with an equilibrium associated to BIS $=50$ given by:

$$
\begin{aligned}
& x_{e 1}=14.51 \mathrm{mg}, \quad x_{e 2}=64.26 \mathrm{mg}, \quad x_{e 3}=809.2 \mathrm{mg}, \\
& x_{e 4}=3.4 \mathrm{mg} / \mathrm{l}, \quad u_{e}=6.08 \mathrm{mg} / \mathrm{min}
\end{aligned}
$$

and parameters $a_{1}=0.5$ and $a_{2}=-0.5$. The parameters used in the output equation (3) are $E C_{50}=3.4 \mathrm{mg} / \mathrm{l}$ and $\gamma=3$ [3], allowing to determine those of the linearized output equation (4): $k_{b i s}=22.06$ and $k_{b i s 0}=125$. With the set of parameters: $\tau_{1}=2.5, \tau_{2}=0.00052, \tau_{3}=0.0055, \tau_{4}=27, \alpha=5.75$, a solution to the optimization problem (54) is given by the controller:

$$
\begin{aligned}
A_{c} & =\left[\begin{array}{cc}
-13.11 & -798.26 \\
0.13 & -24.28
\end{array}\right], B_{c}=\left[\begin{array}{c}
28.31 \\
1.08
\end{array}\right], E_{c}=\left[\begin{array}{l}
-0.89 \\
-0.01
\end{array}\right] \\
C_{c} & =\left[\begin{array}{ll}
13.66 & -194.14
\end{array}\right], D_{c}=18.07 .
\end{aligned}
$$

The analysis of the closed-loop system (14) may then be performed thanks to the optimization procedure (17) (including also the constraint (19)) to build the set $\mathcal{D}$. To build the induction control $u_{I}$, one optimization is performed with the Matlab function fminsearch. With parameters $U_{\min }=-u_{e}$ and $U_{\max }=100 \mathrm{mg} / \mathrm{min}$, and recalling that $\lambda_{1}^{*}(0)$ is known a priori, one obtains:

$$
\lambda^{*}(0)=\left[\begin{array}{llll}
-0.0094 & -0.1876 & 0.0011 & 0.0007
\end{array}\right]
$$

With this initial co-state vector and initial patient state $x_{a n}(0)=0, u_{I}$ turns out to be initially equal to $U_{\max }+u_{e}$ then commutes to 0 at time $T_{\text {com }}=0.545 \mathrm{~min}$, with an optimal $T_{f}=1.842 \mathrm{~min}$.

The patient state trajectory obtained with the switched controller (11) is plotted on Figure 3, with the associated control plotted on Figure 4. The switch between $u_{I}$ and $u_{M}$ occurs as soon as the trajectory enters $\mathcal{D}$. It may be seen in Figure 5, which plots the time evolution of the function $X^{\prime} \epsilon N^{-1} X$, that this happens at time $T_{\text {switch }}=1.7507 \mathrm{~min}$ (i.e. $X^{\prime} \epsilon N^{-1} X$ becomes lower than 1 ).

The corresponding evolution of the BIS is plotted on Figure 6. One can check that the BIS trajectory enters the interval $[40,60]$ at time $t=1.1 \mathrm{~min}$. Furthermore, one can observe that the positivity constraints discussed in Section 2.6 are satisfied. 


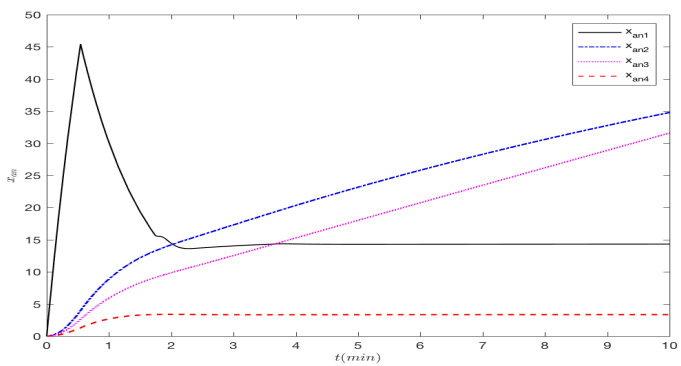

Figure 3: Time evolution of the controlled nominal patient state (components 1 to 4 of state $X$ ).

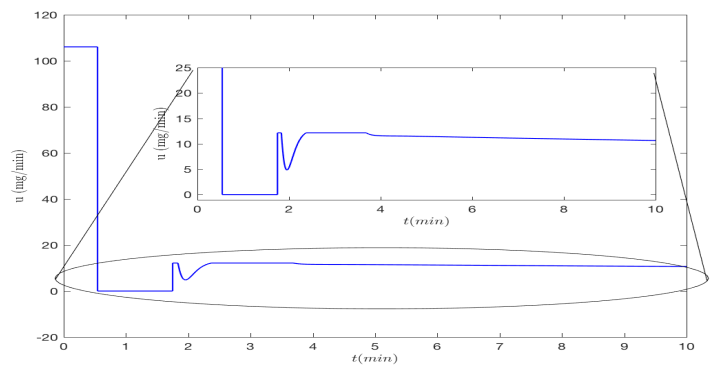

Figure 4: Time evolution of the switched control $u$ ( $u_{I}$ followed by $u_{M}$ as soon as the trajectory enters $\left.\mathcal{D}\right)$.

\section{Robustness issues}

Until now, we have considered an ideal case where the model of a patient is perfectly known. Of course, this is far from being the reality, and we suggest here how the results may be used to approach more realistic situations. Typically, different sources of uncertainty have to be taken into account to go closer to reality.

First of all, the patient model is uncertain, even for a given patient, and the structure itself (4 compartments, Schnider model used to describe the uncertainty), although very frequently used in the literature, should be regarded with caution. Considering the same structure but used to represent a set of patient (the idea being to represent some intra-patient variability as an inter-patient variability), the uncertainty is encapsulated in parameters $k_{10}, k_{12}$ and $k_{21}$. The uncertainties of the dynamics may then be described using an uncertain matrix $A$ included in a polytope with $N=2^{3}=8$ vertices ( 3 being the number of uncertain linear parameters in the matrix), that is:

$$
A=\sum_{i=1}^{N} \lambda_{i} A^{[i]}, \quad \text { with } \sum_{i=1}^{N} \lambda_{i}=1, \quad \lambda_{i} \geq 0
$$

with $A^{[i]}$ corresponding to the vertices of the polytope in which $A$ is defined.

A key property of Proposition 1 is that convexity is preserved when considering a polytopic uncertain matrix $A$. It means that, as soon as conditions (15)-(16) are satisfied for all the vertices $A^{[i]}$, the stability of the uncertain system is satisfied and the resulting set $\mathcal{D}=\mathcal{E}\left(N^{-1}, \epsilon\right)$ is a region of invariance for the uncertain closed-loop system [37]. Sources of uncertainties in the equilibrium (then in parameters $a_{k}$ and $c_{x}$ ) may be addressed in the same way and the optimisation problem (17) may be directly applied for the uncertain case. Moreover, the closed-loop maintenance system is actually globally stable (it can be checked by replacing $Z$ by $N K$ in Proposition 1). It means that one can switch from $u_{I}$ to $u_{M}$ before entering the ellipsoid $\mathcal{D}$ without loosing stability, although to the detriment of the guarantee that the BIS will not pass below 40. Then, as soon as the trajectory will go in $\mathcal{D}$, the closed-loop maintenance control will guarantee that the performance will be satisfied.

On the other hand, the computation of the induction control $u_{I}$ is patient dependent since it has to be performed in open loop. The variability of the patient directly influences the instant of commutation between 


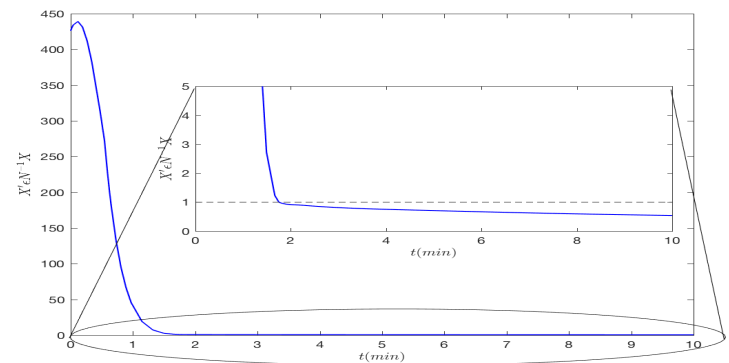

Figure 5: Time evolution of the function $X^{\prime} \in N^{-1} X$. It becomes lower than 1 as soon as the state $X$ enters $\mathcal{D}$.

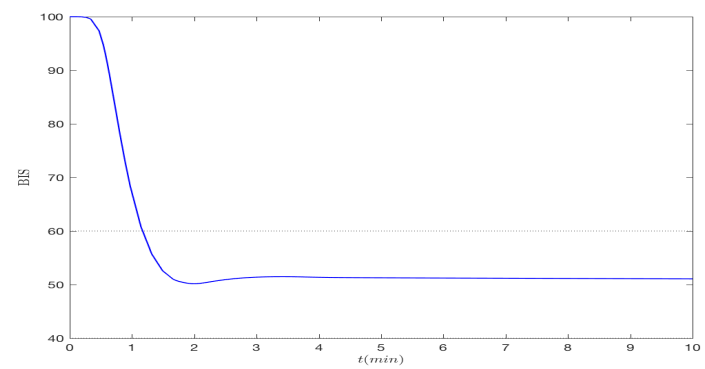

Figure 6: Time evolution of the bispectral index.

$U_{\max }$ and $U_{\min }$. This is not a problem to personalize the induction policy to each patient as the computation of $\lambda^{*}(0)$ (which will induce the commutation) is performed offline, but the intra-patient variability should also be taken into account, at least to guarantee that, although not being time optimal, the open-loop trajectory of the fast state will approach the invariant set $\mathcal{D}$ before to switch.

Let us illustrate those aspects numerically. To evaluate the intra-patient uncertainty, simulations are performed by considering uncertainty of $\pm 10 \%$ on the parameters $k_{10}, k_{12}$ and $k_{21}$, but considering the initial co-state vector $\lambda^{*}(0)$ equal to the solution with the nominal patient (see Section 6). The switch from $u_{I}$ to $u_{M}$ occurs either when entering $\mathcal{D}$ or after 2 minutes. Figure 7 illustrates the simulations with the vertices of the intra-patient uncertainty. One can check that, except for the nominal case (black line), the switch occurs with the time trigger, but this does not prevent the BIS to remain in its safe interval $[40,60]$.

To evaluate now the inter-patient uncertainty, the idea is to consider one single output feedback controller for the maintenance phase, but considering that the induction phase control $u_{I}$ has been adjusted (offline) for each patient. Then if we consider a set of patients, male or female, belonging to interval 20-70 years, $50-100 \mathrm{~kg}$, 150-200 cm, the uncertain pharmacokinetic parameters are given in Table 1.

Table 1: Pharmacokinetic model parameters for the nominal patient (section 6) and for a set of adult patients.

\begin{tabular}{lll}
\hline Parameters & Nominal patient & Set of adult patients \\
\hline$k_{10}\left(\mathrm{~min}^{-1}\right)$ & 0.4191 & {$[0.249,0.898]$} \\
$k_{12}\left(\mathrm{~min}^{-1}\right)$ & 0.3020 & {$[0.206,0.487]$} \\
$k_{21}\left(\mathrm{~min}^{-1}\right)$ & 0.0683 & {$[0.065,0.072]$} \\
$k_{13}\left(\mathrm{~min}^{-1}\right)$ & 0.1958 & 0.1958 \\
$k_{31}\left(\mathrm{~min}^{-1}\right)$ & 0.0035 & 0.0035 \\
\hline
\end{tabular}

From this set of parameters, one can build the eight vertices $A^{[i]}$ of the uncertain dynamics matrix $A$. Then, considering the nominal controller computed in Section 6, the objective is to evaluate the stability of the closed-loop maintenance system. The uncertain version of the optimisation problem (17) allows to verify that the problem is feasible and the ellipsoidal domain obtained in the uncertain case is almost unchanged. Moreover, simulations plotted on Figure 8 allow to illustrate the maintenance control strategy, as soon as the 

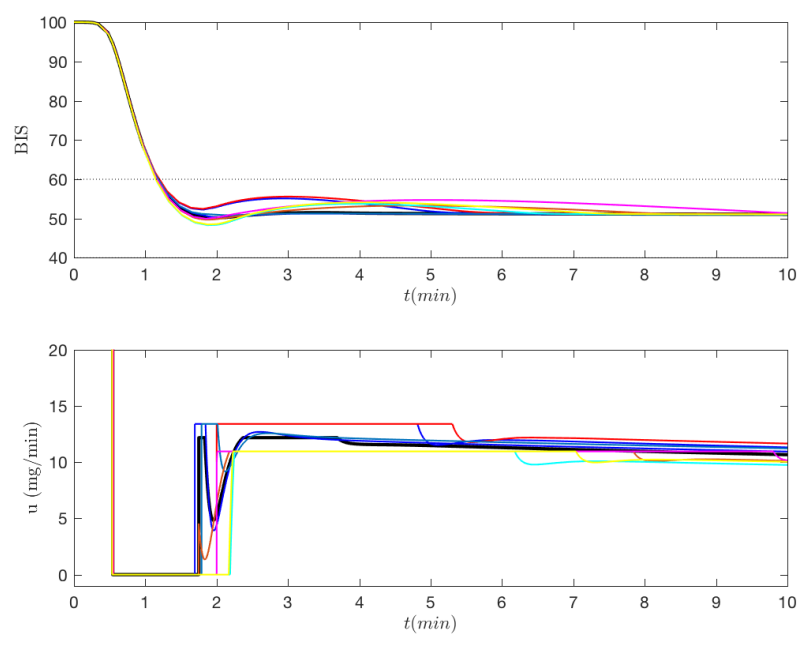

Figure 7: Time evolution of the bispectral index (upper plot) and input $u$ (lower plot) - Uncertain case $( \pm 10 \%$ of variation of parameters $k_{10}, k_{12}$ and $k_{21}$ ). The nominal case is also plotted in solid black line.

optimal control $u_{I}$ is preliminary computed for each patient (eventually with some intra-patient uncertainty).
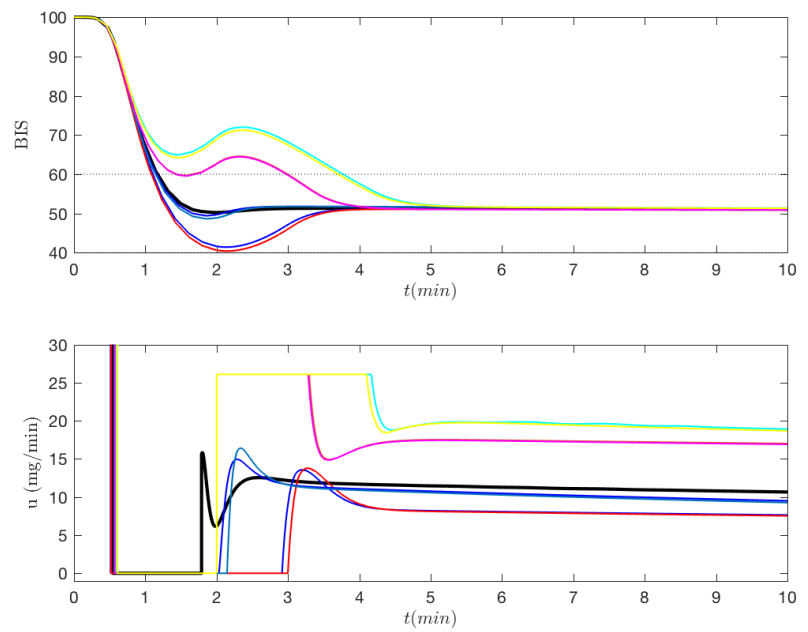

Figure 8: Time evolution of the bispectral index (upper plot) and input $u$ (lower plot) - Uncertain case representing the inter-patient variability described in Table 1.

One can also check that, for the nominal controller computed in Section 6, the closed-loop dynamics is subject to the influence of uncertainties on the closed-loop maintenance control system. Typically, the slow modes are almost unchanged (as expected), and the modes issued from the controller are also the same around $\{-15 \pm 4\}$. On the other hand, the two modes related to the fast dynamics significantly vary between $\{-2.78,-7.36\}$ and $\{-3.80 \pm 1.72\}$ for the set of patients described in Table 1 .

Another source of uncertainty could be taken into account. Although not very often explicitly considered in the literature, the presence of a delay in the relationship between the site effect concentration and the BIS indicator has been underlined by several authors [30,18]. Just to illustrate the importance of taking into account the delay, one can check in Figure 9 that increasing delay may induce instability: the system remains stable with 10 seconds of delay but becomes instable with 30 seconds of delay. During the induction phase, the control is 
essentially open loop and, then, is not affected by such a measurement delay. During the maintenance phase, delay may be considered with a delay-version of Proposition 1 (see [15] for example, which could be expanded to the case with fast-slow dynamics).
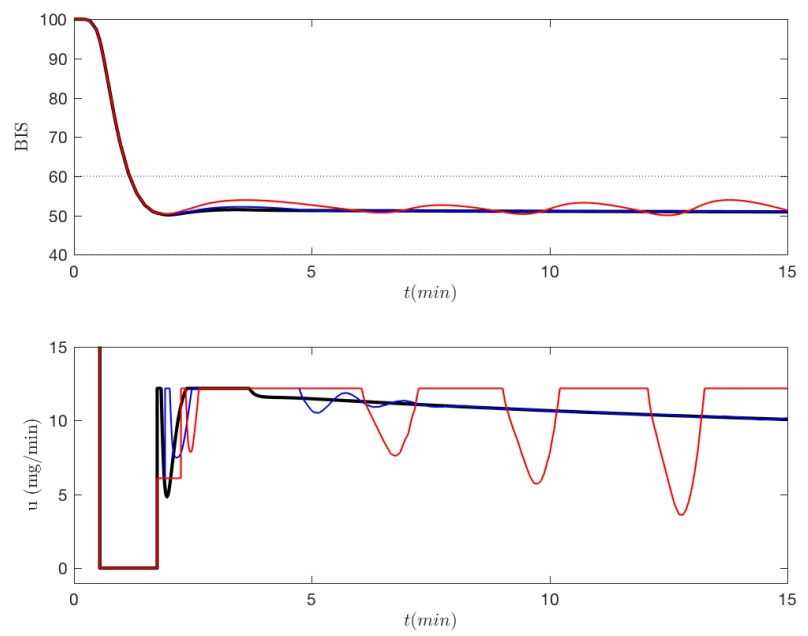

Figure 9: Time evolution of the bispectral index (upper plot) and input $u$ (lower plot) - Influence of an output delay.

\section{Conclusion}

In this paper, we have shown how to construct a switching control strategy inspired by medical practice. Indeed, using an optimal control scheme, we mimic the strategy already considered by the anesthesiologist with an initial injection of a large quantity of drug (bolus) for a short time, followed by a phase without addition of drugs $\left(u_{a n}=0\right)$ before switching to the maintenance phase. Only the open-loop phase may be considered as optimal. As soon as the trajectory enters the invariant ellipsoidal domain, the controller switches to the closed-loop maintenance phase and it is no more question of time optimality but of regulation. This type of result can be also recovered by using the invariant set theory (see, for example, the developments in the discrete-time case in [14]). The stability of the overall scheme is guaranteed when the switch occurs in a safe invariant domain of the closed-loop maintenance system. The approach considers a nominal problem where the patient model is known (related to its physical characteristics as suggested by [32]) but the extension towards more realistic situations is discussed. Of course, this paper does not claim to solve the real-life control problem, but intends rather to give significant insights to approach it, with a switched control rule combining an optimal open-loop action to induce anesthesia and a output feedback control to maintain hypnosis of the patient.

The results proposed in the paper imply several directions for future works. In particular, one could consider the reference tracking problem and address it through the addition of an integrator in the control loop or thanks to the use of more advanced techniques as that one issued from reset control systems. Furthermore, in order to be closer to the real-life anesthesia, control scheme with sampled output or even event-triggered output controller could be investigated. Finally, further works could more explicitly consider some key properties of anesthesia dynamics, that are singularly perturbed systems and positive systems, in a combined control design strategy.

\section{References}

[1] A. R. Absalom and G. N C. Kenny. Closed-loop control of propofol anaesthesia using bispectral index: Performance assessment in patients receiving computer-controlled propofol and manually controlled remifentanil infusions for minor surgery. British Journal of Anaesthesia, 90:737-741, 2003. 
[2] M. Athans and P. L. Falb. Optimal control: an introduction to the theory and its applications. Courier Corporation, 2013.

[3] J. M. Bailey and W. M. Haddad. Drug dosing control in clinical pharmacology. IEEE Control Systems Magazine, 25(2):35-51, 2005.

[4] C. L. Beck. Modeling and control of pharmacodynamics. European Journal of Control, 24:33-49, 2015.

[5] A. Berman, M. Neumann, and R. J. Stern. Nonnegative Matrices in Dynamic Systems. Wiley-Interscience, John Wiley and Sons, New York, USA, 1989.

[6] I. Biswas, P.J. Mathew, R.S. Singh, and G.D. Puri. Evaluation of closed-loop anesthesia delivery for propofol anesthesia in pediatric cardiac surgery. Pediatric Anesthesia, 23(12):1145-1152, 2013.

[7] F. Cacace, L. Farina, R. Setola, and A. Germani (Eds). Positive systems - Theory and Applications (POSTA 2016), volume 471 of LectureNotesinControlandInformationSciences. Springer, 2017.

[8] M. J. Coppens, D. J. Eleveld, J. H. Proost, L. A. Marks, J. F. Van Bocxlaer, H. Vereecke, A. R. Absalom, and M. M. Struys. An evaluation of using population pharmacokinetic models to estimate pharmacodynamic parameters for propofol and bispectral index in children. Anesthsiology, 115(1):83-93, 2011.

[9] G. C. Cummings, J. Dixon, N. H. Kay, J. P. W. Windsor, E. Major, M. Morgan, J. W. Sear, A. A. Spence, and D. K. Stephenson. Dose requirements of ici 35,868 (propofol, 'diprivan') in a new formulation for induction of anaesthesia. Anaesthesia, 39(12):1168-1171, 1984.

[10] H. Derendorf and B. Meibohm. Modeling of pharmacokinetic / pharmacodynamic (pk/pd) relationships: Concepts and perspectives. Pharmaceutical Research, 16(2):176-185, 1999.

[11] G.A. Dumont, A. Martinez, and J.M. Ansermino. Robust control of depth of anesthesia. International Journal of Adaptive Control and Signal Processing, 23(5):435-454, 2009.

[12] C. Dussaussoy, M. Peres, V. Jaoul, N. Liu, T. Chazot, J. Picquet, M. Fischler, and L. Beydon. Automated titration of propofol and remifentanil decreases the anesthesiologist's workload during vascular or thoracic surgery: a randomized prospective study. Journal of clinical monitoring and computing, 28(1):35-40, 2014.

[13] Y. Ebihara. $H_{2}$ analysis of LTI systems via conversion to extrernally positive systems. IEEE Transactions on Automatic Control, To appear.

[14] M. Fiacchini, I. Queinnec, S. Tarbouriech, and M. Mazerolles. Invariant based control of induction and maintenance phases for anesthesia. In 6th IFAC Conference on Foundations of Systems Biology in Engineering, Magdeburg, Germany, 2016.

[15] J.M. Gomes da Silva Jr, I. Ghiggi, and S. Tarbouriech. Non-rational dynamic output feedback for time-delay systems with saturating inputs. International Journal of Control, 81(4):557-570, 2008.

[16] W. M. Haddad, V. S. Chellaboina, and Q. Hui. Nonnegative and compartmental dynamical systems. Princeton University Press, 2010.

[17] W. M. Haddad, T. Hayakawa, and J. M. Bailey. Adaptive control for non-negative and compartmental dynamical systems with applications to general anesthesia. International Journal of Adaptive Control and Signal Processing, 17(3):209-235, 2003.

[18] C.M. Ionescu, R. Hodrea, and R. De Keyser. Variable time-delay estimation for anesthesia control during intensive care. IEEE Transactions on Biomedical Engineering, 58(2):363-369, 2011.

[19] M. Le Guen, N. Liu, F. Tounou, M. Augé, O. Tuil, T. Chazot, D. Dardelle, P.-A. Laloë, F. Bonnet, D.I. Sessler, et al. Dexmedetomidine reduces propofol and remifentanil requirements during bispectral index-guided closed-loop anesthesia: a double-blind, placebo-controlled trial. Anesthesia 83 Analgesia, 118(5):946-955, 2014. 
[20] J. M. Lemos, D. V. Caiado, B. A. Costa, L. A. Paz, T. F. Mendonca, S. Esteves, and M. Seabra. Robust control of maintenance-phase anesthesia. IEEE Control Systems Magazine, 34(6):24-38, December 2014.

[21] D. Liberzon. Switching in systems and control. Birkhäuser, 2003.

[22] D. Liberzon. Calculus of variations and optimal control theory: a concise introduction. Princeton University Press, 2012.

[23] N. Liu, T. Chazot, S. Hamada, A. Landais, N. Boichut, C. Dussaussoy, B. Trillat, L. Beydon, E. Samain, D.I. Sessler, and M. Fischler. Closed-loop coadministration of propofol and remifentanil guided by bispectral index: a randomized multicenter study. Anesthesia $\&$ Analgesia, 112(3):546-557, 2011.

[24] D. C. Mackey. Can We Finally Conquer the Problem of Medical Quality? Anesthesiology, 117(2):225-226, 2012.

[25] M. Martins da Silva, T. Wigren, and T. Mendonça. Nonlinear identification of a minimal neuromuscular blockade model in anesthesia. IEEE Transactions on Control Systems Technology, 20(1):181-188, 2012.

[26] D. S. Naidu. Optimal control systems. CRC press, 2002.

[27] I. Nascu, A. Krieger, C. M. Ionescu, and E. N. Pistikopoulos. Advanced model based control studies for the induction and maintenance of intravenous anaesthesia. IEEE Transactions on Biomedical Engineering, 62(3):832-841, February 2015.

[28] F.N. Nogueira, T. F. Mendonca, and P. Rocha. Controlling the depth of anesthesia by a novel positive control strategy. Computer methods and programs in Biomedicine, 114:e87-e97, 2014.

[29] C. Rocha, T. Mendonça, and M.E. Silva. Individualizing propofol dosage: a multivariate linear model approach. Journal of clinical monitoring and computing, 28(6):525-536, 2014.

[30] Y. Sawaguchi, E. Furutani, G. Shirakami, M. Araki, and K. Fukuda. A model-predictive hypnosis control system under total intravenous anesthesia. IEEE Transactions on Biomedical Engineering, 55(3):874-887, 2008.

[31] C. Scherer, P. Gahinet, and M. Chilali. Multiobjective output-feedback control via LMI optimization. IEEE Transactions on Automatic Control, 42:896-911, 1997.

[32] T. W. Schnider, C. F. F Minto, P. L. Gambus, C. Andresen, D. B. Goodale, S. L. Shafer, and E. J. Youngs. The influence of method of administration and covariates on the pharmacokinetics of propofol in adult volunteers. Anesthesiology, 88(5):1170-1182, 1998.

[33] Z. Shu, J. Lam, H. Gao, B. Du, and L. Wu. Positive observers and dynamic output-feedback controllers for interval positive linear systems. IEEE Transactions on Circuits and Systems - I, 55(10):3209-3222, November 2008.

[34] K. Soltész. On Automation in Anesthesia. PhD thesis, Lund University, Sweden, 2013.

[35] M M Struys, T De Smet, L F Versichelen, S Van De Velde, R Van den Broecke, and E P Mortier. Comparison of closed-loop controlled administration of propofol using Bispectral Index as the controlled variable versus "standard practice" controlled administration. Anesthesiology, 95(1):6-17, 2001.

[36] S. Syafiie, M. Ait Rami, and F. Tadeo. Positive infusion of propofol drug during induction. In IEEE International Conference on Industrial Engineering and Engineering Management, pages 325-328, 2010.

[37] S. Tarbouriech, G. Garcia, J. M. Gomes Da Silva Jr, and I. Queinnec. Stability and Stabilization of Linear Systems with Saturating Actuators. Springer, 2011.

[38] K. Van Heusden, G.A. Dumont, K. Soltesz, C.L. Petersen, A. Umedaly, N. West, and J.M. Ansermino. Design and clinical evaluation of robust PID control of propofol anesthesia in children. IEEE Transactions on Control Systems Technology, 22(2):491-501, 2014. 
[39] S. Zabi, I. Queinnec, G. Garcia, S. Tarbouriech, and M. Mazerolles. Time-optimal control for the induction phase of hypnosis during anesthesia. Control Engineering Practice, 2018. (submitted).

[40] S. Zabi, I. Queinnec, S. Tarbouriech, G. Garcia, and M. Mazerolles. New approach for the control of anesthesia based on dynamics decoupling. In 9th IFAC Symposium on Biological and Medical Systems (BMS 2015), Berlin, Germany, 2015.

[41] L. Zaccarian and A.R. Teel. Modern Anti-windup Synthesis: Control Augmentation for Actuator Saturation: Control Augmentation for Actuator Saturation. Princeton Series in Applied Mathematics. Princeton University Press, 2011.

[42] X.-S. Zhang and R. J. Roy. Derived fuzzy knowledge model for estimating the depth of anesthesia. IEEE Transactions on Biomedical Engineering, 48(3):312-323, 2001. 